\title{
Molecular Dynamics Simulations of Diffuse Scattering
}

\author{
David Wych*, Michael E. Wall ${ }^{* *}$, David Mobley* \\ *University of California Irvine, Irvine, CA \\ ** Los Alamos National Laboratory, Los Alamos, NM \\ Email Contact: dwych@uci.edu
}

For decades, X-ray crystallography has been an indispensable tool for the elucidation of atomic-detail small-molecule and protein structure. In addition to the information contained in the sharp, high-intensity Bragg Peaks, X-ray diffraction patterns also contain weaker, more dispersed areas of intensity around and away from the Bragg Peaks known as "diffuse scattering." While the Bragg Peaks can be processed to reproduce the average electron density in the unit cell (a static picture), the diffuse intensity contains information about the motions of the proteins or small molecules in the crystal, and correlations in those motions. However, methods for processing or interpreting the diffuse intensity are less well developed.

Many models have been proposed to characterize and interpret the information contained in the protein X-ray crystallographic diffuse scattering. Examples include liquid-like lotions (LLM) models ${ }^{1}$, rigid-body motion models (TLS ${ }^{2}$, rigid-body rotation and translation models ${ }^{3}$ ), elastic network/normal-modes models ${ }^{4}$, and conformational ensemble models ${ }^{5}$. While many of these models are reasonably successful at reproducing anisotropic features of the diffuse intensity, they fail to accurately reproduce the isotropic profile of the diffuse intensity.

Molecular dynamics models simulate protein crystals and the surrounding solvent environment using all-atom structures and force-fields which determine their dynamics. These models have shown promise $^{6}$ in reproducing both the isotropic and anisotropic profiles of the diffuse intensity; however, the accuracy is still limited compared to what is needed to validate detailed models of molecular motions.

The aim of our work is to investigate the degrees of freedom that are most important in determining the accuracy of molecular dynamics models of protein crystallographic diffuse scattering, and to gain insights into how to improve model accuracy. So far, we have investigated various choices with respect to the forcefield (AMBER 14SB and CHARMM 27), crystal solvation procedure, solvent/mother liquor reproductions, and protein termini models. The consequences of these choices on the accuracy of the resulting diffuse scattering intensity predictions will be discussed.

1. Wall, M. E., Clarage, J. B. \& Phillips, G. N. (1997). Structure, 5, 1599-1612.

2. Van Benschoten, A. H., Afonine, P. V., Terwilliger, T. C., Wall, M. E., Jackson, C. J., Sauter, N. K., Adams, P. D., Urzhumtsev, A. \& Fraser, J. S. (2015). Acta Cryst. D71, 1657-1667.

3. de Klijn, T., Schreurs, A. M. M. \& Kroon-Batenburg, L. M. J. (2019). IUCrJ 6, 277-289.

4. Riccardi, D., Cui, Q. \& Phillips, G. N. Jr (2010). Biophys. F. 99, 2616-2625.

5. Guinier, A. (1963). X-ray Diffraction in Crystals, Imperfect Crystals, and Amorphous Bodies. San

Francisco: W. H. Freeman \& Co.

6. Wall ME, et al. (2014). Proc. Natl. Acad. Sci. USA 111(50):17887-17892 